\title{
Systematic Review of Breast-Q: A Tool to Evaluate Post-Mastectomy Breast Reconstruction
}

\author{
Ishith Seth' \\ Nimish Seth ${ }^{2}$ \\ Gabriella Bulloch ${ }^{3}$ \\ Warren M Rozen ${ }^{4}$ \\ David J Hunter-Smith ${ }^{4}$ \\ 'Department of Surgery, Bendigo Health, \\ Bendigo, Victoria, 3550, Australia; \\ ${ }^{2}$ Department of Surgery, The Alfred \\ Hospital, Melbourne, Victoria, 3004, \\ Australia; ${ }^{3}$ Faculty of Science, Medicine \\ and Health, University of Melbourne, \\ Melbourne, Victoria, 3010, Australia; \\ ${ }^{4}$ Peninsula Clinical School, Central \\ Clinical School at Monash University, The \\ Alfred Centre, Melbourne, Victoria, \\ 3004, Australia
}

Correspondence: David J Hunter-Smith Peninsula Clinical School, Central Clinical School at Monash University, The Alfred

Centre, 99 Commercial Road,

Melbourne, Victoria, 3004, Australia

Tel +610359763522

Fax +610359763544

Email dhuntersmith@mac.com
Purpose: The aim of this systematic review is to update and synthesize new evidence on BREAST-Q questionnaire's ability to reflect patient-reported outcomes in women who have undergone breast reconstruction surgery (BRS) following mastectomy.

Methods: PubMed, Science Direct, Google Scholar, Cochrane CENTRAL, and Clincaltrial. gov were searched for relevant studies from January 2009 to September 2021. Any interventional or observational studies that used BREAST-Q to assess patient-reported outcomes in the assessment of BRS following mastectomy were included.

Results: A total of 42 studies were eligible for inclusion in the review. Three were randomized controlled trials and 39 were observational studies. Compared with pre-operative scores, there was an improvement in all BREAST-Q outcome domains following BRS including 'satisfaction with breasts', "satisfaction with outcome" "psychosocial", "physical", and "sexual wellbeing". Sexual well-being had the lowest BREAST-Q score both pre-and post-operatively (37.8-80.0 and 39.078.0, respectively). Autologous BRS reports higher satisfaction and overall wellbeing compared to implant-based BRS. BREAST-Q has a higher and narrow internal consistency of 0.81 to 0.96 compared with other patient-reported outcome measures (PROMs; EORTC-QLQ, FACT-B, BR23, BCTOS). The BREAST-Q questionnaire is the only PROM which allows patients to reflect on their care, surgical outcomes, and satisfaction collectively.

Conclusion: This review highlights the fact that BREAST-Q can effectively and reliably measure satisfaction and wellbeing of breast cancer patients after BRS. Comparatively, sexual wellbeing shows poorer outcomes following BRS and more longitudinal studies are necessary to understand the basis for these findings. Compared to other PROMs, BREAST-Q is reliable and specific to breast cancer surgery. Overall, BREAST-Q can help clinicians improve their quality of service, understand patient experiences, and may be used as an auditing tool for surgical outcomes.

Keywords: BREAST-Q, patient-reported outcomes, breast reconstruction surgery, mastectomy

\section{Introduction}

Breast cancer is the most prevalent type of cancer globally. In 2020 alone 2.3 million women were diagnosed with breast cancer worldwide, and 7.8 million women are currently living with it. ${ }^{1}$ Over $30 \%$ of these women undergo a single mastectomy, ${ }^{2,3}$ or prophylactic double mastectomy. ${ }^{4}$ For many, the loss of one or both breasts is devastating, and breast reconstruction surgery (BRS) can improve outcomes for these patients. ${ }^{5,6}$ Over $40 \%$ of women who undergo mastectomy opt for a BRS. ${ }^{7}$

Surgical management strategies for breast cancer may involve mastectomy, breast conservative surgery, BRS, and other reconstructive methods. ${ }^{8-10}$ Age, body habitus, 
co-morbidities, previous surgeries, and other neo-adjuvant treatment influence the surgical method of choice. ${ }^{11,12}$ Many of the women opting for BRS are often eligible for more than one type of breast reconstruction, therefore the timing of reconstruction, use of autologous tissue versus implants, short-versus long-term outcomes, and financial implications are all factors a patient may contemplate. ${ }^{9}$

Patient-reported outcomes (PROs) have become increasingly important in health care and assess the perception of health, quality of life (QoL), and functional status after treatment. ${ }^{13}$ In cosmetic/reconstructive surgery, this is particularly important as the aim of the intervention is often to improve appearance, function, mental health, and QoL. ${ }^{13}$ These tools can also help patients become informed, form realistic expectations, communicate with the surgical team, and gain greater satisfaction from the decision-making process. ${ }^{14,15}$

Patient-reported outcome measures (PROMs) are tools used to quantify PROs, often in the form of self-completed questionnaires. ${ }^{16}$ The BREAST-Q is a PROM used to assess the unique outcomes of breast surgery patients. ${ }^{17}$ Developed in 2009, BREAST-Q is made up of three procedure-specific modules: augmentation, reduction, and reconstruction. ${ }^{18}$ The questionnaire examines outcomes commonly reported as important to women who have undergone a reconstructive procedure for breast cancer as well as health-related quality of life (HRQoL), psychosocial, physical, and sexual well-being, and satisfaction scales. ${ }^{17}$ Since its development, BREAST-Q has been an effective measure for a spectrum of breast cancer surgeries. ${ }^{19,20}$

While several studies have used the BREAST-Q to assess the outcomes of patients undergoing breast surgeries for breast cancer, only one comprehensive systematic review exists on PROMs assessed by BREAST-Q which is now outdated and had heterogeneous results. ${ }^{19}$ Hence, our review aims to update and synthesize new evidence on BREAST-Q's ability to reflect PROs in women who have undergone BRS following mastectomy. This review will address the following questions:

- To what extent has BREAST-Q evaluated PROM amongst patients who have undergone BRS?

- What were the outcome parameters used for BREAST-Q?

- How does BREAST-Q compare to other available PROMs?
- Is BREAST-Q an effective tool for measuring PROMs in BRS?

\section{Methods}

\section{Search Strategy}

This review adhered to the Preferred Reporting in Systematic Review \& Meta-Analysis (PRISMA) guidelines and was listed retrospectively on the PROSPERO International Prospective Register of Systematic Review (CRD42021278102). ${ }^{21}$ PubMed, Google Scholar, Science Direct, Cochrane CENTRAL, and trial registries (http:// clinicialtrials.gov/) were searched for relevant studies published from January 1st, 2009 to September 30th, 2021. The search terms included: ("mastectomy" OR "breast removal surgery") AND ("breast reconstructive surgery" OR "BRS") AND ("BREAST-Q" OR "BREAST-Q questionnaire"). Furthermore, the references of selected articles were manually searched for relevant articles. After a screening of titles and abstracts, full-text reports were assessed for eligibility.

\section{Study Selection and Outcomes}

Inclusion criteria:

1. Primary research published in peer-reviewed journals including experimental such as randomized control trials (RCTs) and non-randomized trials, and observational such as cohort and case-control studies;

2. Studies with a target population included women with primary breast cancer who had mastectomy, or women who had prophylactic mastectomy. No restrictions were placed on age, type, and stage of breast cancer;

3. Studies reporting outcomes of any type of BRS following mastectomy;

4. Studies reporting outcomes using BREAST-Q.

Exclusion criteria:

1. Studies not published in English language:

2. Reviews, pre-prints, case reports, conference proceedings, conference abstracts, and letters or editorial opinions.

3. Studies on breast cancer in general without specific reference to BRS.

4. Studies that did not use BREAST-Q questionnaire as a PROM, or did not fully report BREAST-Q 
satisfaction or health-related quality of life outcomes.

\section{Data Collection and Extraction}

Titles and abstracts of studies identified during the search were imported into Endnote X9 (https://endote.com) for preliminary screening. Full texts of potentially relevant papers were further screened using the eligibility criteria. These were done by two independent reviewers (IS and GB), and any disparity in either selecting eligible articles or assessing findings between the two reviewers was resolved through consultation with a third reviewer (NS). The following data were extracted from each included article into a pre-structured data collection sheet: year of publication, the country where the study was done, sample size, average age, the type of BRS, follow-up period, outcomes measured by BREAST-Q (such as reliability, and responsiveness), and average BREAST-Q scores.

\section{Quality Assessment}

The methodological quality of each study will be assessed using the Cochrane Systematic Review RCT risk of bias assessment tool 2 (RoB 2) for RCTs, ${ }^{22,23}$ and Joanna Briggs Institute (JBI) Critical Appraisal Checklist for observational studies. ${ }^{24}$ The RoB 2 tool addresses the following biases: random sequence generation, bias due to deviations from intended interventions, bias due to incomplete outcome data, bias in measurement of the outcome, and selective reporting. The items were assessed as "low risk", "high risk", or "some concerns". The JBI is a reliable and valid tool used to assess the methodological quality of observational cross-sectional studies. The checklist consists of eight questions, with 4 possible answers: 'yes', "no", "unclear", and "not applicable". A final question on whether to include, exclude, or seek further information on the study gives the overall judgement of the reviewer on that study. Disagreements were resolved through discussion with a third reviewer.

\section{Data Synthesis}

Data extracted from included articles were analyzed and combined in a narrative synthesis. Information from the studies were coded based on their methodologies and findings. These were then organized into subheadings and descriptive categories. Tables and charts were used to present the results. Through study screening, there was a wide variation of BRS types evident in literature, therefore a meta-analysis would show significant heterogeneity and ungeneralizable results.

\section{Results}

\section{Study Characteristics}

A total of 719 studies were identified from the literature search, and 43 met the eligibility criteria (Figure 1). All included studies identified their target population as patients who had a therapeutic or prophylactic mastectomy for breast cancer. The sample sizes ranged from 22 to 2048. Nineteen studies reported on implant-based BRS, 9 reported on autologous BRS, and 15 reported on both. One study did not include the type of procedure carried out on subjects. Most studies were conducted in the United States (13), with other countries being Italy (7), United States and Canada (4), Canada only (3), Netherlands (4), Germany (3), Mexico (1), Portugal (1), India (1), Australia (1), Denmark (1), United Kingdom (1) and Czech Republic (1). Studies were published between 2009 and 2021. Only three studies used a randomized controlled study design, while others used an observational design. Fifteen studies were of retrospective design while 28 were of prospective design. The average age ranged from 43.3 to 67 years, and the average follow-up duration at the time of administration of BREAST-Q tool ranged from 1 month to 6.5 years (Table 1).

\section{Patient Reported Outcomes Using BREAST-Q}

All studies used the breast reconstruction module of the BREAST-Q tool and aimed to assess the satisfaction and/ or QoL of participants after BRS following mastectomy. All but one study reported the respondents' BREAST-Q scores on "satisfaction with breast" and "satisfaction with outcome" were reported by 31 studies. Wellbeing, psychosocial wellbeing, sexual wellbeing, and physical wellbeing (chest and upper body) were reported by 39, 39, and 37 studies, respectively. Thirteen studies assessed satisfaction with information, surgeon, medical team, and administrative staff domains, respectively.

On the BREAST-Q tool, the scoring for each domain ranges from 0 , signifying the least possible level of satisfaction or wellbeing, to 100, signifying the highest. The average scores on satisfaction with breast domain ranged from 39.5 to 75.8 pre-operatively and $51.1-82.0$ post-operatively while satisfaction with overall outcome ranged from 56.3 to 89 . Average psychosocial well-being scores ranged from 54.3 to 


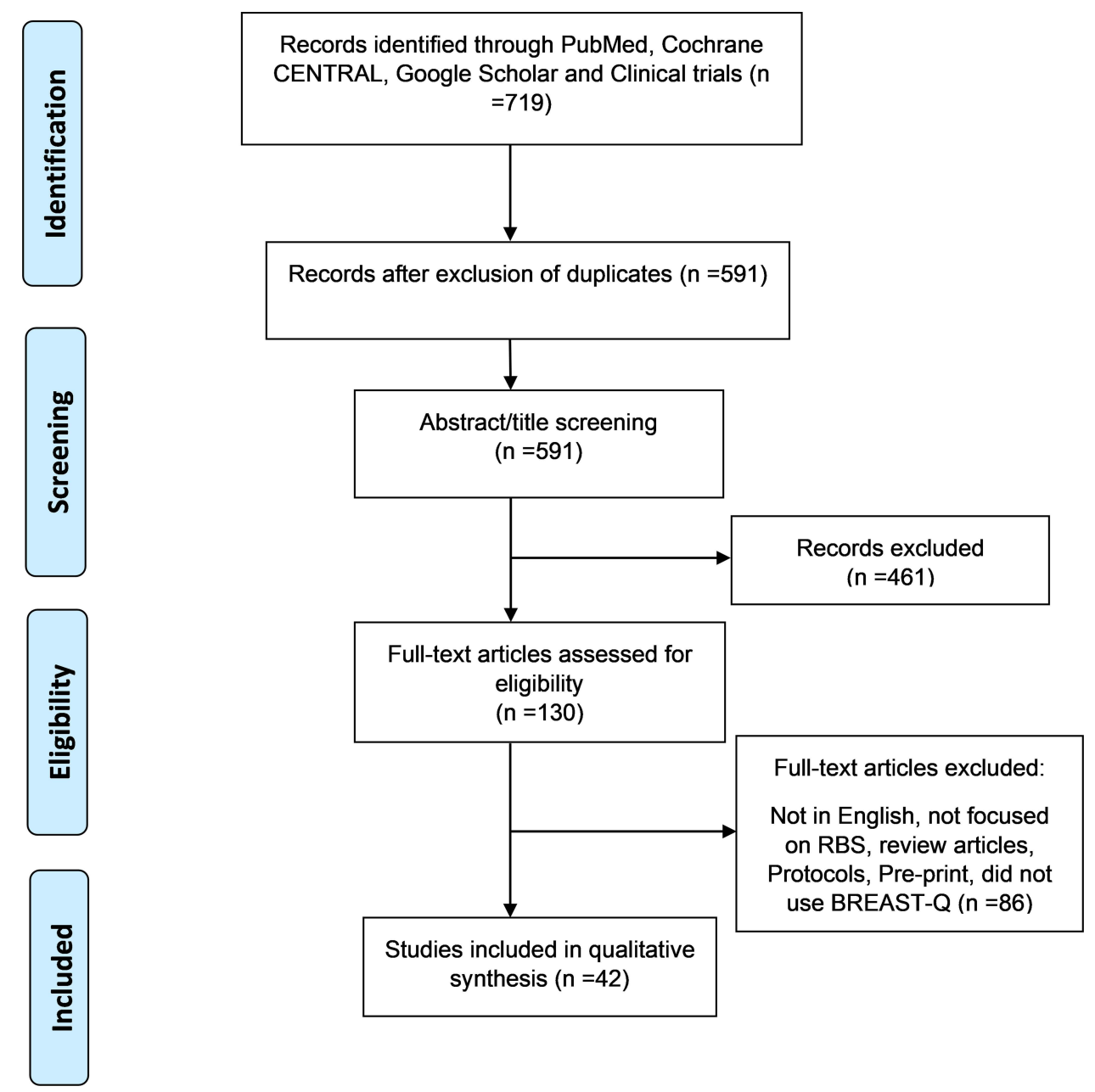

Figure I PRISMA flow diagram of selected studies.

77.9 on pre-operative assessment and $63.0-94.0$ on postoperative assessment. Sexual well-being had the lowest average scores in all the studies, ranging from 37.8 to 80.0 on pre-operative assessment and 39.0-78.0 on postoperative assessment. Physical well-being (chest and upper body) had average scores ranging from 57.8 to 81.4 at baseline and 53.2-83.0 post-operatively. The studies also reported high satisfaction rates with medical care. Average scores for satisfaction with information ranged from 53.5 to 89, satisfaction with the surgeon, 83.2-100.0, Satisfaction with the medical team, 78.0-100.0, and satisfaction with the administrative team, 81.5-100.0.

\section{BREAST-Q Response Rate}

Of the 42 studies, only 15 reported the response rate for completion of the BREAST-Q questionnaire, which ranged from $38.4 \%$ to $98 \%$ (Figure 2).

\section{Comparison Between Different BRS Types}

Most studies compared PROs between different types of BRS. Comparisons were based on timing (immediate versus delayed), ${ }^{25-27}$ type (implant-based versus autologous), ${ }^{28-37}$ type of flap used (deep inferior epigastric perforators [DIEP], transverse rectus abdominis myocutaneous [TRAM], latissimus dorsi [LD] flaps), ${ }^{38-40}$ type of Implant/tissue expanders used (acellular dermal matrices [ADM], silicone expanders, saline expanders), ${ }^{41-44}$ placement of implant (pre-pectoral versus sub-pectoral), ${ }^{45,46}$ number of stages (single stage versus multiple stages), ${ }^{25-27,45}$ weight (normal weight, overweight, obese) ${ }^{47}$ and age (less than 60 versus over 60 , less than 65 versus over 65$).{ }^{39,48}$

\section{Autologous vs Implant-Based BRS}

In nearly all studies that compared PROs between autologous and implant-based BRS, autologous BRS had better 
Table I Characteristics of Included Studies

\begin{tabular}{|c|c|c|c|c|c|c|}
\hline $\begin{array}{l}\mathbf{S} / \\
\mathbf{N}\end{array}$ & $\begin{array}{l}\text { First } \\
\text { Author } \\
\text { (Year) }\end{array}$ & Country & Study Type & $\begin{array}{l}\text { Sample } \\
\text { Size }\end{array}$ & Average Age (Years) ${ }^{a}$ & Type of Breast Reconstruction \\
\hline I. & $\begin{array}{l}\text { Stein et al } \\
(2020)^{4 I}\end{array}$ & Canada & RCT & 62 & $\begin{array}{l}\text { Alloderm group: } 49 \text { (12.2); } \\
\text { Dermacell group: } 54(9.6)\end{array}$ & $\begin{array}{l}\text { Direct-to-implant and two-staged } \\
\text { pectoral BRS }\end{array}$ \\
\hline 2. & $\begin{array}{l}\text { Casella et al } \\
(2019)^{26}\end{array}$ & Italy & Retrospective & 397 & $46.5(13.6)$ & ADM and non-ADM tissue expanders \\
\hline 3. & $\begin{array}{l}\text { Sorkin et al } \\
(2017)^{42}\end{array}$ & $\begin{array}{l}\text { United } \\
\text { States }\end{array}$ & Prospective & 1297 & $48.4(10.4)$ & Autologous BRS \\
\hline 4. & $\begin{array}{l}\text { Sinha et al } \\
(2016)^{47}\end{array}$ & Australia & Prospective & 101 & $\begin{array}{l}\text { Normal weight: } 46 \text {; overweight: } \\
\text { 49; and obese patients: } 49\end{array}$ & $\begin{array}{l}\text { IBBRS versus autologous. Fat grafting } \\
\text { versus no fat grafting }\end{array}$ \\
\hline 5. & $\begin{array}{l}\text { Bennett et al } \\
(20 \mid 7)^{28}\end{array}$ & $\begin{array}{l}\text { United } \\
\text { States }\end{array}$ & Prospective & 2048 & $49.4(10)$ & Autologous \\
\hline 6 & $\begin{array}{l}\text { Song et al } \\
(2016)^{48}\end{array}$ & $\begin{array}{l}\text { United } \\
\text { States, } \\
\text { Canada }\end{array}$ & Retrospective & 1809 & $\begin{array}{l}\text { Patients <65years: } 49 \text {; patients } \\
>65 \text { years: } 67\end{array}$ & Autologous, IBBRS and mixed \\
\hline 7. & $\begin{array}{l}\text { Davis et al } \\
(2014)^{29}\end{array}$ & $\begin{array}{l}\text { United } \\
\text { States }\end{array}$ & Retrospective & 134 & Median(range): 49(19-66) & $\begin{array}{l}\text { Free TRAM flap (autologous) and } \\
\text { IBBRS }\end{array}$ \\
\hline 8 & $\begin{array}{l}\text { Pirro et al } \\
(2017)^{30}\end{array}$ & $\begin{array}{l}\text { Czech } \\
\text { Republic }\end{array}$ & Prospective & 65 & $\begin{array}{l}\text { TRAM group: 51.2; Implant group: } \\
58.9\end{array}$ & Autologous, IBBRS and Mixed \\
\hline 9. & $\begin{array}{l}\text { Klifto et al } \\
(2020)^{31}\end{array}$ & $\begin{array}{l}\text { United } \\
\text { States }\end{array}$ & Retrospective & 600 & $\begin{array}{l}\text { Control(normal): } 43.3(15.2) \text {; } \\
\text { BRS group: 49.9(9.4) }\end{array}$ & $\begin{array}{l}\text { Direct-to-implant and Implant/tissue } \\
\text { expander reconstruction }\end{array}$ \\
\hline 10. & $\begin{array}{l}\text { Srinivasa et al } \\
(2017)^{27}\end{array}$ & $\begin{array}{l}\text { United } \\
\text { States }\end{array}$ & Prospective & 1427 & $48.4(10.4)$ & IBBRS and autologous \\
\hline II. & $\begin{array}{l}\text { Santosa et al } \\
(2016)^{32}\end{array}$ & $\begin{array}{l}\text { United } \\
\text { States }\end{array}$ & Prospective & 1531 & NR & $\begin{array}{l}\text { Pectoral Implant placement with } \\
\text { TiLoop }^{\circledR} \text { bra-mesh }\end{array}$ \\
\hline 12. & $\begin{array}{l}\text { Casella et al } \\
(2018)^{43}\end{array}$ & Italy & Prospective & 179 & $56.3(23-79)$ & NR \\
\hline 13. & $\begin{array}{l}\text { Ranieri et al } \\
(202 \mathrm{I})^{72}\end{array}$ & Italy & Prospective & 44 & $40.4(5.9)$ & $\begin{array}{l}\text { Autologous: latissimus dorsi flap versus } \\
\text { thoracodorsal artery perforator flap }\end{array}$ \\
\hline 14. & $\begin{array}{l}\text { Rindom et al } \\
(2020)^{38}\end{array}$ & Denmark & RCT & 40 & NR & $\begin{array}{l}\text { Autologous reconstruction versus } \\
\text { IBBRS }\end{array}$ \\
\hline 15. & $\begin{array}{l}\text { Reinders et al } \\
(2019)^{33}\end{array}$ & Netherlands & $\begin{array}{l}\text { Retrospective } \\
\text { and } \\
\text { prospective }\end{array}$ & 112 & $\begin{array}{l}\text { Autologous: } 43(8.0) \text {; IBBRS: } 49.4 \\
(10.1)\end{array}$ & Autologous versus IBBRS \\
\hline 16. & $\begin{array}{l}\text { Albornoz } \\
\text { et al }(2014)^{58}\end{array}$ & $\begin{array}{l}\text { United } \\
\text { States, } \\
\text { Canada }\end{array}$ & Prospective & 633 & $\begin{array}{l}\text { Irradiated: } 52.0(10.8) \text {; non- } \\
\text { irradiated: } 50.5(9.2)\end{array}$ & IBBRS with versus without irradiation \\
\hline 17. & $\begin{array}{l}\text { Koppiker et al } \\
(2018)^{59}\end{array}$ & India & Retrospective & 78 & NR & Autologous: DIEP versus TRAM flaps \\
\hline 18. & $\begin{array}{l}\text { Ludolph et al } \\
(2015)^{39}\end{array}$ & Germany & Retrospective & 179 & $\begin{array}{l}\text { Patients<60years: } 48.2 \text {; } \\
\text { patients>60years: } 63.8\end{array}$ & Autologous \\
\hline
\end{tabular}


Table I (Continued).

\begin{tabular}{|c|c|c|c|c|c|c|}
\hline $\begin{array}{l}\mathbf{S} / \\
\mathbf{N}\end{array}$ & $\begin{array}{l}\text { First } \\
\text { Author } \\
\text { (Year) }\end{array}$ & Country & Study Type & $\begin{array}{l}\text { Sample } \\
\text { Size }\end{array}$ & Average Age (Years) ${ }^{a}$ & Type of Breast Reconstruction \\
\hline 19. & $\begin{array}{l}\text { Allen et al } \\
(2020)^{73}\end{array}$ & $\begin{array}{l}\text { United } \\
\text { States }\end{array}$ & Prospective & 405 & $50.1(7.9)$ & IBBRS versus autologous \\
\hline 20. & $\begin{array}{l}\text { Santosa et al } \\
(2018)^{34}\end{array}$ & $\begin{array}{l}\text { United } \\
\text { States }\end{array}$ & Prospective & 2013 & $\begin{array}{l}\text { IBRS: 48.I(I0.5); Autologous: } 51.6 \\
(8.7)\end{array}$ & IBBRS \\
\hline 21. & $\begin{array}{l}\text { Koslow et al } \\
(2013)^{74}\end{array}$ & $\begin{array}{l}\text { United } \\
\text { States }\end{array}$ & Retrospective & 294 & $\begin{array}{l}\text { No contralateral prophylactic } \\
\text { mastectomy (CPM): } 50.2 ; \\
\text { CPM: } 54.7\end{array}$ & IBBRS and autologous \\
\hline 22. & $\begin{array}{l}\text { Klement et al } \\
(2019)^{35}\end{array}$ & $\begin{array}{l}\text { United } \\
\text { States }\end{array}$ & Retrospective & 96 & $\begin{array}{l}\text { Median (range) } \\
\text { Implant: 49(27-74); Flap: } \\
\text { immediate-5I(26-68), delayed- } \\
50.5(32-7 \mathrm{I})\end{array}$ & Autologous \\
\hline 23. & $\begin{array}{l}\text { Menez et al } \\
(2017)^{75}\end{array}$ & Italy & Retrospective & 98 & 51.7 & Autologous: DIEP flap \\
\hline 24. & $\begin{array}{l}\text { Razzano et al } \\
(2018)^{76}\end{array}$ & NR & Prospective & 70 & $55(8.6)$ & $\begin{array}{l}\text { Silicone implants and TiLoop }{ }^{\circledR} \text { bra } \\
\text { mesh }\end{array}$ \\
\hline 25. & $\begin{array}{l}\text { Casella et al } \\
(2018)^{43}\end{array}$ & Italy & $\begin{array}{l}\text { Prospective } \\
\text { longitudinal }\end{array}$ & 46 & $43.2(23-65)$ & Autologous: DIEP and TRAM flap \\
\hline 26. & $\begin{array}{l}\text { Zhong et al } \\
(201 \mathrm{II})^{40}\end{array}$ & Canada & Prospective & 55 & Median(range): 48(28-77) & IBBRS: saline and silicone implants \\
\hline 27. & $\begin{array}{l}\text { Macadam et al } \\
(2010)^{44}\end{array}$ & Canada & Retrospective & 143 & $\begin{array}{l}\text { Saline implant: } 55.6(9.1) \text {; silicone: } \\
52.3(9.5)\end{array}$ & Autologous tissue and IBBRS \\
\hline 28. & $\begin{array}{l}\text { Martinez- } \\
\text { Lopez et al } \\
(2021)^{36}\end{array}$ & Mexico & Retrospective & 153 & $56(14.2)$ & TRAM, Latissimus dorsi flap and IBBRS \\
\hline 29. & $\begin{array}{l}\text { Brito et al } \\
(2020)^{37}\end{array}$ & Portugal & Prospective & 284 & $48.8(9.0)$ & IBBRS-saline and silicone \\
\hline 30. & $\begin{array}{l}\text { McCarthy } \\
\text { et al }(2010)^{49}\end{array}$ & $\begin{array}{l}\text { Canada, } \\
\text { United } \\
\text { States }\end{array}$ & Prospective & 520 & $\begin{array}{l}\text { Saline: } 51.3(10.4) \text {; silicone: } 53.7 \\
\text { (II.0) }\end{array}$ & TRAM flap, expander/implant, \\
\hline 31. & $\begin{array}{l}\text { Hu et al } \\
(2009)^{77}\end{array}$ & $\begin{array}{l}\text { United } \\
\text { States }\end{array}$ & Retrospective & 342 & $\begin{array}{l}\text { Median(range) } \\
\text { Expander: 52.9(19-79); } \\
\text { TRAM:52.3(34-72) }\end{array}$ & IBBRS and autologous \\
\hline 32. & $\begin{array}{l}\text { Pusic et al } \\
(2017)^{78}\end{array}$ & $\begin{array}{l}\text { United } \\
\text { States, } \\
\text { Canada }\end{array}$ & Prospective & 1183 & $49.9(9.9)$ & IBBRS \\
\hline 33. & $\begin{array}{l}\text { Negenborn } \\
\text { et al }(2018)^{50}\end{array}$ & Netherlands & Retrospective & 208 & $43.2(10.1)$ & $\begin{array}{l}\text { IBBRS with/without opposite breast } \\
\text { reduction }\end{array}$ \\
\hline 34. & $\begin{array}{l}\text { Shekhawat } \\
\text { et al }(2015)^{79}\end{array}$ & India & Prospective & 147 & Median(range): 48(29-72) & IBBRS alone and IBBRS with mesh \\
\hline
\end{tabular}

(Continued) 
Table I (Continued).

\begin{tabular}{|c|c|c|c|c|c|c|}
\hline $\begin{array}{l}\mathbf{S} / \\
\mathbf{N}\end{array}$ & $\begin{array}{l}\text { First } \\
\text { Author } \\
\text { (Year) }\end{array}$ & Country & Study Type & $\begin{array}{l}\text { Sample } \\
\text { Size }\end{array}$ & Average Age (Years) ${ }^{a}$ & Type of Breast Reconstruction \\
\hline 35. & $\begin{array}{l}\text { Dieterich } \\
\text { et al }(2015)^{80}\end{array}$ & Germany & Retrospective & 61 & $\begin{array}{l}\text { IBBRS alone: } 52.8(9.4) ; \text { IBBRS with } \\
\text { mesh: } 49.4(8.4)\end{array}$ & $\begin{array}{l}\text { Direct-to-implant, tissue expander/ } \\
\text { implant }\end{array}$ \\
\hline 36. & $\begin{array}{l}\text { Qureshi et al } \\
(2017)^{25}\end{array}$ & $\begin{array}{l}\text { United } \\
\text { States }\end{array}$ & Prospective & 59 & $44(I I)$ & Autologous, mesh \\
\hline 37. & $\begin{array}{l}\text { Sewart et al } \\
(2020)^{81}\end{array}$ & $\begin{array}{l}\text { United } \\
\text { Kingdom }\end{array}$ & Prospective & 891 & Median(range) 50(45-58) & Autologous and IBBRS \\
\hline 38. & $\begin{array}{l}\text { Eltahir et al } \\
(2014)^{82}\end{array}$ & Netherlands & Retrospective & 92 & $\begin{array}{l}\text { Median(range) Autologous: } 5 \text { I } \\
\text { (35-78); Implant: } 44.0(26.62)\end{array}$ & DIEP flap \\
\hline 39. & $\begin{array}{l}\text { Ochoa et al } \\
(2018)^{83}\end{array}$ & $\begin{array}{l}\text { United } \\
\text { States }\end{array}$ & Prospective & 73 & Median (range): $5 \mathrm{I}(22-73)$ & $\begin{array}{l}\text { IBBRS with pre-pectoral silicone } \\
\text { implants }\end{array}$ \\
\hline 40. & $\begin{array}{l}\text { Spindler et al } \\
(2021)^{84}\end{array}$ & Germany & Prospective & 22 & Median(range): 40.II(28-58) & Direct-to-implant \\
\hline 41. & $\begin{array}{l}\text { Caputo et al } \\
(2020)^{45}\end{array}$ & Italy & Retrospective & 94 & Subpectoral-53; prepectoral:53 & $\begin{array}{l}\text { IBBRS with ADM and two-staged } \\
\text { IBBRS }\end{array}$ \\
\hline 42 & $\begin{array}{l}\text { Negenborn } \\
\text { et al }(2018)^{61}\end{array}$ & Netherlands & RCT & 142 & $\begin{array}{l}\text { IBBRS with ADM: } 43.5(I I .6) \text { and } \\
\text { Two-staged IBBRS: } 47.4(I 2.2)\end{array}$ & $\begin{array}{l}\text { Direct-to-implant and two-stages } \\
\text { pectoral BRS }\end{array}$ \\
\hline 43 & $\begin{array}{l}\text { Ghilli et al } \\
(2019)^{46}\end{array}$ & Italy & Prospective & 132 & 48.88 & $\begin{array}{l}\text { Autologous: } \\
\text { subpectoral prosthesis and ADM, pre- } \\
\text { pectoral prosthesis and TiLoop }{ }^{\circledR} \text { mesh }\end{array}$ \\
\hline
\end{tabular}

Notes: ${ }^{a}$ Mean (standard deviation) except otherwise stated.

Abbreviations: ADM, acellular dermal matrices; BRS, breast reconstruction surgery; IBBRS, implant-based breast reconstruction surgery; NR, not reported; RCT, randomized controlled trial; TRAM, transverse rectus abdominis myocutaneous; DIEP, deep inferior epigastric perforators.

outcomes comparatively. ${ }^{28-37}$ Table 2 shows the average BREAST-Q scores for the HRQoL subscales between autologous and implant-based BRS. In all domains, autologous BRS had higher post-operative scores compared to implant-based.

\section{Type of Flaps and Implants Used}

Only two studies in this review compared the PROs following autologous BRS with different flap types. Rindom et al compared the PROs between BRS with a latissimus dorsi (LD) flap and a thoracodorsal artery perforator flap, while Ludolph et al compared the PROs between DIEP and TRAM. ${ }^{38,39}$ These two studies found no significant difference between the two groups in respect to all satisfaction and HRQoL domains, as both groups reported high satisfaction rates. ${ }^{38,39}$ Similarly, two studies compared the use of saline and silicone implants. Both found that silicone implants showed better PROs compared to saline implants. $^{44,49}$ Sorkin et al found no difference between the use of $\mathrm{ADM}$ and non-ADM tissue expanders the PROs of patients. ${ }^{42}$

\section{Single-Stage versus Multiple-Stage BRS}

Negenborn et al and Qureshi et al found no significant differences in all BREAST-Q domains between patients who underwent one-stage BRS and those who underwent two-stages implant BRS using tissue expanders. ${ }^{25,50}$ Another study found no significant difference in the PROs of patients in both direct-to-implant (DTI) group and tissue expander groups, except in sexual wellbeing, where the DTI group fared better. ${ }^{27}$

\section{BREAST-Q versus Other PROMs}

The BREAST-Q tool was compared with five other HRQoL PROM questionnaires (Table 3). BREAST-Q covers a wide range of domains compared with other PROMs and is the only tool that assesses individuals' satisfaction with care received. While all tools generally reported good 


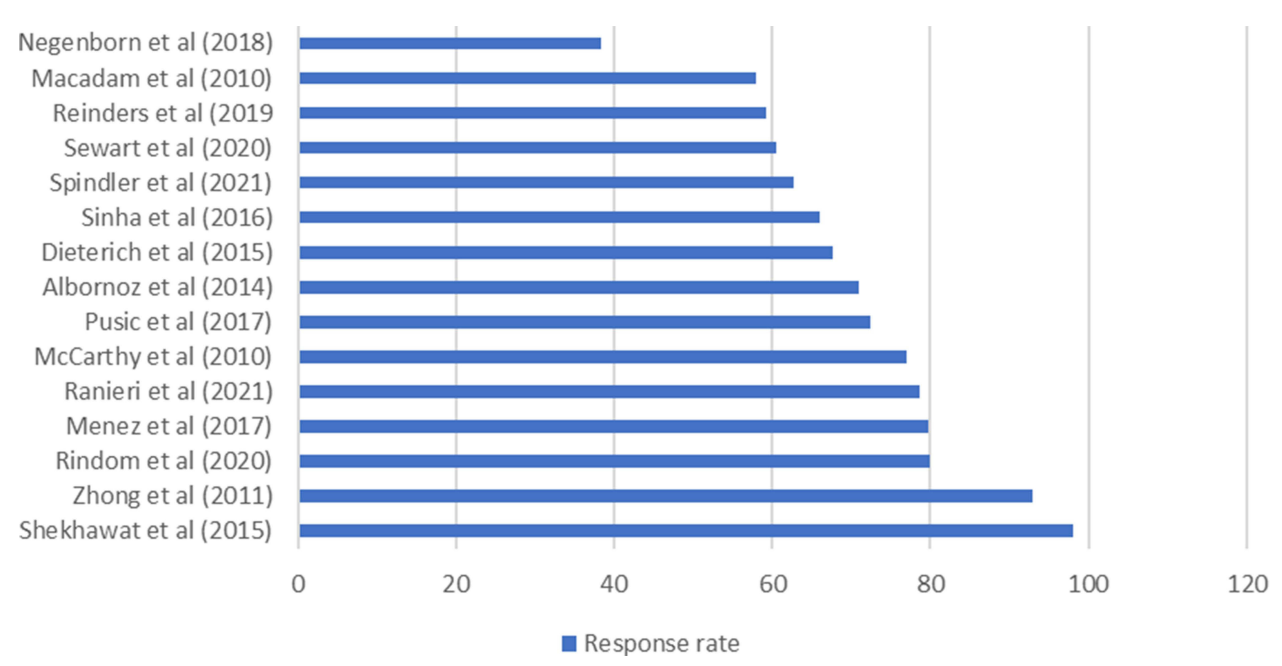

Figure 2 Response rate for completion of BREAST-Q questionnaire (\%).

internal consistency/reliability with Rasch analysis, a statistical tool that assesses psychometric properties, BREAST-Q had a narrower range of reliability $(0.81-$ 0.96 , compared to $0.69-0.9 .0$ and $0.46-0.91$ as seen with EORTC QLQ 30 and BR-23 respectively) and is considered psychometrically robust. ${ }^{17,51,52}$ The test re-test reproducibility of BREAST-Q ranges from 0.73 to $0.96 .{ }^{17}$ Only the BREAST-Q reconstruction module is specific to HRQoL after BRS following mastectomy.

\section{Methodological Quality of Studies}

Of the included studies, only 3 were RCTs, and all had low risk of bias (Figure 3). The JBI Critical Appraisal Checklist was used to assess the methodological quality of the observational studies. All studies were of high quality and were therefore included in the review.

\section{Discussion}

The BREAST-Q questionnaire is a validated tool for evaluating PROs in patients undergoing BRS following mastectomy. Generally, BRS using either autologous or implant-based methods resulted in greater satisfaction and HRQoL. This review also found that autologous BRS had better PROs than implant-based BRS in all BREAST-Q domains. No statistical differences were noticed between the different types of flaps studied, however patients with silicone implants had better BREAST-Q scores indicating greater satisfaction and HRQoL. Patients that underwent one-staged and two-staged breast reconstructions fared similarly.

Alongside subjective outcomes, patient satisfaction is an indicator of surgical success and predicts psychosocial health following BRS. Within the included studies, average BREAST-Q scores for "satisfaction with breasts" domain ranged from 39.5 to 75.8 pre-operatively and increased to 51.1-82.0 post-operatively which can be attributed to a favorable change in body image. In contrast, satisfaction amongst patients who underwent mastectomy without BRS was poorer, with women being unhappy with their breasts and surgical scar despite the cancer being successfully treated. ${ }^{53,54}$ Duggal et al found that over three-quarters of their participants opting for BRS had body image as their motivating factor. ${ }^{55}$ These BREAST-Q scores and supporting findings suggest BRS should be indicated for patients who house concerns about body image, or hope to improve body image following mastectomy.

Table 2 Average BREAST-Q Score for Different Breast Reconstruction Surgeries Across Included Studies. Each Domain Ranges from 0, Signifying the Least Possible Level of Satisfaction or Wellbeing, to 100, Signifying the Highest (0-100 Range in Each Domain)

\begin{tabular}{|l|l|l|l|l|l|}
\hline $\begin{array}{l}\text { Type of Breast } \\
\text { Reconstruction Surgery }\end{array}$ & $\begin{array}{c}\text { Satisfaction with } \\
\text { Breast }\end{array}$ & $\begin{array}{c}\text { Satisfaction with } \\
\text { Results }\end{array}$ & $\begin{array}{c}\text { Psychosocial Well } \\
\text { Being }\end{array}$ & $\begin{array}{c}\text { Sexual Well } \\
\text { Being }\end{array}$ & $\begin{array}{c}\text { Physical Well- } \\
\text { Being }\end{array}$ \\
\hline Autologous & 70.3 & 78.9 & 77.2 & 59.9 & 76.7 \\
\hline Implant-based & 63.8 & 72.9 & 77.2 & 59.9 & 76.7 \\
\hline
\end{tabular}


Table 3 Comparison of BREAST-Q Questionnaire with Other Patient-Reported Outcome Questionnaires

\begin{tabular}{|c|c|c|c|c|}
\hline $\begin{array}{l}\text { Patient-Reported } \\
\text { Outcome Measures } \\
\text { (PROMs) Questionnaires }\end{array}$ & Domains Assessed & $\begin{array}{l}\text { PROMs } \\
\text { Range }\end{array}$ & Psychometric Analysis (Reliability) & $\begin{array}{l}\text { Specificity } \\
\text { to Breast } \\
\text { Cancer }\end{array}$ \\
\hline BREAST-Q & $\begin{array}{l}9 \text { domains: } \\
\text { - physical well-being } \\
\text { - psychosocial well-being } \\
\text { - sexual well-being } \\
\text { - satisfaction with breast } \\
\text { - satisfaction with outcome } \\
\text { - satisfaction with information } \\
\text { - satisfaction with surgeon } \\
\text { - satisfaction with medical team } \\
\text { - satisfaction with admin staff }\end{array}$ & $\begin{array}{l}0-100 \text { in } \\
\text { each } \\
\text { domain }\end{array}$ & $\begin{array}{l}\text { (HRQoL after BRS) } \\
\text { Internal consistency (Cronbach's alpha) ranges } \\
\text { from } 0.81 \text { to } 0.96 ; ;^{17,51,52} \text { Test re-test } \\
\text { reproducibility } 0.73 \text { to } 0.96^{17}\end{array}$ & $\begin{array}{l}\text { Specific to } \\
\text { BRS following } \\
\text { mastectomy }\end{array}$ \\
\hline EORTC-Q30 & $\begin{array}{l}5 \text { items on the functional scale: } \\
\text { - physical, role, social, emotional, and cognitive } \\
\text { functioning } \\
9 \text { items on the symptom scale: } \\
\text { - pain, fatigue, financial impact, appetite loss, } \\
\text { nausea/vomiting, diarrhea, constipation, sleep } \\
\text { disturbance and quality of life }\end{array}$ & $\begin{array}{l}30 \text { items: } 0 \\
100 \text { in each } \\
\text { domain }\end{array}$ & $\begin{array}{l}\text { (HRQoL in cancer) } \\
\text { Internal consistency (Cronbach's alpha) ranges } \\
\text { from } 0.69-0.9^{85,86}\end{array}$ & $\begin{array}{l}\text { Not specific } \\
\text { to breast } \\
\text { cancer }\end{array}$ \\
\hline EORTC QLQ BR-23 & $\begin{array}{l}5 \text { items on the functional scale: } \\
\text { - physical, role, social, emotional, and cognitive } \\
\text { functioning } \\
4 \text { items on the symptom scale: } \\
\text { - systemic therapeutic side effect, breast } \\
\text { symptoms, arm symptoms, upset by hair loss }\end{array}$ & $\begin{array}{l}23 \text { items: } 0 \\
100 \text { in each } \\
\text { domain }\end{array}$ & $\begin{array}{l}\text { (HRQoL after nreast cancer treatment) Internal } \\
\text { consistency (Cronbach's alpha) ranges from } \\
0.46-0.91^{85,87,88}\end{array}$ & $\begin{array}{l}\text { Specific to } \\
\text { breast cancer }\end{array}$ \\
\hline Short-Form 36 & $\begin{array}{l}8 \text { domains: } \\
\text { - physical functioning } \\
\text { - physical role limitations } \\
\text { - bodily pain } \\
\text { - general health perceptions } \\
\text { - energy/vitality } \\
\text { - social functioning } \\
\text { - emotional role limitations } \\
\text { - mental health }\end{array}$ & $\begin{array}{l}36 \text { items: } 0 \\
100 \text { in each } \\
\text { domain }\end{array}$ & $\begin{array}{l}\text { (General HRQoL) Internal consistency } \\
\text { (Cronbach's alpha) ranges from } 0.72-0.91^{89-91}\end{array}$ & $\begin{array}{l}\text { Not specific } \\
\text { to cancer }\end{array}$ \\
\hline $\begin{array}{l}\text { FACT-B@ David Cella, } \\
1987,1997\end{array}$ & $\begin{array}{l}5 \text { domains: } \\
\text { - physical, social, emotional, functional well- } \\
\text { being } \\
\text { a breast-cancer subscale: } \\
\text { - shortness of breath } \\
\text { - self-consciousness about the way I dress } \\
\text { - one or both of my arms are swollen or tender } \\
\text { - I feel sexually attractive } \\
\text { - I am bothered by hair loss } \\
\text { - I worry that other members of my family might } \\
\text { someday get the same illness I have } \\
\text { - I worry about the effect of stress on my illness } \\
\text { - I am bothered by a change in weight } \\
\text { - I am able to feel like a woman } \\
\text { - I have certain parts of my body where } \\
\text { I experience pain }\end{array}$ & 37 items & $\begin{array}{l}\text { (HRQoL after breast cancer treatment) Internal } \\
\text { consistency (Cronbach's alpha) ranges from } \\
0.70-0.90^{92-94}\end{array}$ & $\begin{array}{l}\text { Specific to } \\
\text { breast cancer }\end{array}$ \\
\hline вCTOS & $\begin{array}{l}3 \text { domains: } \\
\text { - functional status, cosmetic status, and breast- } \\
\text { specific pain }\end{array}$ & 22 items & $\begin{array}{l}\text { (HRQoL after breast cancer treatment) Internal } \\
\text { consistency (Cronbach's alpha) ranges from } \\
0.81-0.91^{95} \text {. }\end{array}$ & $\begin{array}{l}\text { Specific to } \\
\text { breast cancer }\end{array}$ \\
\hline
\end{tabular}

Abbreviations: BCTOS, breast cancer treatment outcome scale; BRS, breast reconstruction surgery; EORTC, European organization for research and treatment of cancer; FACT-B, functional assessment of cancer therapy - breast; HRQoL, health-related quality of life. 


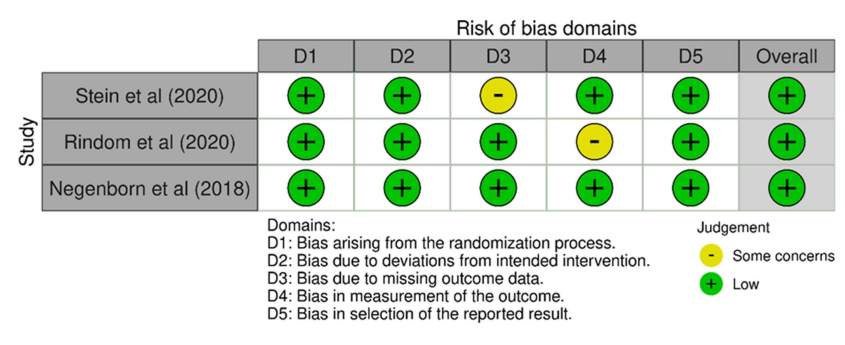

Figure 3 Risk of bias assessment for included RCTs.

The reviewed literature suggests BREAST-Q can indicate which BRS will yield greatest outcomes in satisfaction (Tables 1 and 2). All types of BRS yielded improvements with breast satisfaction following surgery and continued to improve over time except for Stein et al and Negenborn et al, who notably used ADM alongside tissue expanders/implant BRS. ${ }^{41,50}$ In these surgeries, lower satisfaction with breast/s, overall outcome, physical and sexual wellbeing outcomes following BRS were observed. ${ }^{41,50}$ A possible explanation for these findings is that ADM is associated with higher post-operative complications including seroma, infections, and red breast syndrome which may affect patient QoL and satisfaction. $^{41,50,56,57}$ Another factor that worsened BREAST-Q scores was radiotherapy, which is also associated with higher rates of complications in autologous and implant-based BRS. ${ }^{33,58,59}$ Knowing the difference in complication rates in these BRS cohorts would better explain the low level of satisfaction observed.

Physical and psychosocial wellbeing following BRS was assessed in most of the included studies and showed overall improvement. An exception to this was Rowland et al who found patients undergoing mastectomy with and without reconstruction showed declined physical wellbeing, but this was equal when compared with women undergoing lumpectomy. ${ }^{60}$ Another domain, sexual wellbeing, generally decreased following mastectomy compared with pre-operative BREAST-Q scores, and after BRS there was reportedly worsened to minimal improvements. ${ }^{41,42,61}$ Overall, sexual wellbeing fared the least compared to all other domains which can be explained by the psychological effects of breast cancer surgery which can include anxiety, depression, and a feeling of loss of femininity. ${ }^{62}$ Moreover, pain and discomfort in the months following surgery may impact the pursuit or desire for sexual activity. In the latter case, patient sexual well-being should improve following complete healing which could take up to a year or more. ${ }^{63}$
Future longitudinal studies are needed to define the etiology of this domain because if decreased sexual wellbeing is due to mental health, this defines an opportunity to address it with a health professional.

The BREAST-Q questionnaire also allows patients to reflect on their relationship with the surgeon, the information that they received, and the care provided by the administrative staff. ${ }^{17}$ Although most studies did not assess this domain, the ones that did show patients had high levels of satisfaction with the care that they received. More studies should focus on this PRO because these measures can be used to monitor and improve quality of care by surgeons and hospitals, and for auditing by health governance. Satisfaction with care has also been known to influence other outcome domains such as "satisfaction with breasts" and physical wellbeing including HRQoL. In future, this PRO should be focused upon more and be viewed as a potentially valuable tool for measuring quality of care. ${ }^{64}$

The BREAST-Q questionnaire was designed to measure outcomes which should be examined in BRS. ${ }^{65,66}$ When examined by Rasch analysis, BREAST-Q has a high narrow internal consistency and test-retest reproducibility. ${ }^{17,51,52}$ This strongly supports that it is valid and reliable tool for its purpose. Other PROMs used in assessing HRQoL in breast cancer patients are equally reliable but have wide reported range. In contrast to these questionnaires, BREAST-Q is also specific to BRS and is the only tool to accurately assess patient satisfaction with care (Table 3). ${ }^{67,68}$ Chen et al reported BREAST-Q as one of the best tools for assessing HRQoL in breast cancer patients, stating that it was able to address surgery-specific issues, unlike other PROMs. ${ }^{69}$ In support, the International Consortium for Health Outcomes Measurement endorsed BREAST-Q for breast cancer patients, highlighting its approval by healthcare governing bodies for assessing oncoplastic BRS outcomes. ${ }^{70}$

The current systematic review achieved its aims to examine the current evidence about BREAST-Q for management of post-mastectomy BRS and was able to compare it with the other PROMs (Table 3). Furthermore, the current study adopted a well-structured search strategy, followed the PRISMA guidelines and utilized manual searches to identify most eligible studies, and only included studies that were of good methodological quality. Despite these benefits, this study has several limitations. Firstly, the BREAST-Q tool collects self-reported data which can be unreliable; however, for subjective outcomes self-reported data are an accepted measure of choice. ${ }^{71}$ Secondly, the level of heterogeneity in 
BRS procedures prevented the performance of a metaanalysis and pooled analysis. Lastly, there was diversity amongst the geographic origin of included studies which may have introduced sociocultural factors. The impact of these would need to be statistically explored in metaanalysis, however the heterogeneity between studies made a meta-analysis unsuitable.

\section{Conclusion}

This review highlights that BREAST-Q can effectively and reliably measure satisfaction and HRQoL of breast cancer patients after BRS. Comparatively, sexual wellbeing shows poorer outcomes following BRS and more longitudinal studies are necessary to understand the basis for these findings. Overall, BREAST-Q can help clinicians improve their quality of service, understand patient experiences, and may be used as an auditing tool for surgical outcomes.

\section{Author Contributions}

All authors made a significant contribution to the work reported, whether that is in the conception, study design, execution, acquisition of data, analysis and interpretation, or in all these areas; took part in drafting, revising, or critically reviewing the article; gave final approval of the version to be published; have agreed on the journal to which the article has been submitted; and agree to be accountable for all aspects of the work.

\section{Funding}

This research did not receive any specific grant from funding agencies in the public, commercial, or not-forprofit sector. The authors have no financial relationships relevant to this article to disclose.

\section{Disclosure}

The authors have no conflicts of interest to disclose.

\section{References}

1. World Health Organization. Breast cancer [Internet]; 2020 [cited September 5, 2021.]. Available from: https://www.who.int/news-room /fact-sheets/detail/breast-cancer. Accessed November 29, 2021.

2. Harding C, Pompei F, Burmistrov D, Wilson R. Use of mastectomy for overdiagnosed breast cancer in the United States: analysis of the SEER 9 cancer registries. J Cancer Epidemiol. 2019;2019:e5072506.

3. Dragun AE, Huang B, Tucker TC, Spanos WJ. Increasing mastectomy rates among all age groups for early stage breast cancer: a 10-year study of surgical choice. Breast J. 2012;18(4):318-325.

4. Dragun AE, Pan J, Riley EC, et al. Increasing use of elective mastectomy and contralateral prophylactic surgery among breast conservation candidates: a 14-year report from a comprehensive cancer center. Am J Clin Oncol. 2013;36(4):375-380.
5. Heidari M, Shahbazi S, Ghodusi M. Evaluation of body esteem and mental health in patients with breast cancer after mastectomy. J Midlife Health. 2015;6(4):173.

6. Arroyo JMG, López MLD. Psychological problems derived from mastectomy: a qualitative study. Int J Surg Oncol. 2011;4(2011):e132461.

7. Ilonzo N, Tsang A, Tsantes S, Estabrook A, Thu MAM. Breast reconstruction after mastectomy: a ten-year analysis of trends and immediate postoperative outcomes. Breast. 2017;32:7-12.

8. Bellavance EC, Kesmodel SB. Decision-making in the surgical treatment of breast cancer: factors influencing women's choices for mastectomy and breast conserving surgery. Front Oncol. 2016;29(6):74.

9. Sun CS, Cantor SB, Reece GP, Fingeret MC, Crosby MA, Markey MK. Helping patients make choices about breast reconstruction: a decision analysis approach. Plast Reconstr Surg. 2014;134 (4):597-608.

10. Fingeret MC, Nipomnick SW, Crosby MA, Reece GP. Developing a theoretical framework to illustrate associations among patient satisfaction, body image and quality of life for women undergoing breast reconstruction. Cancer Treat Rev. 2013;39(6):673-681.

11. Cutress RI. Oncoplastic breast reconstruction: guidelines for best practice [Internet]. In: Rainsbury D, Willett A, editors. British Association of Plastic Reconstructive and Aesthetic Surgeons; 2012: 68.[cited September 5, 2021]: Available from: https://eprints.soton.ac. uk/345578/. Accessed November 29, 2021.

12. Schmauss D, Machens H-G, Harder Y. Breast reconstruction after mastectomy. Front Surg. 2015;2:71.

13. Weldring T, Smith SMS. Patient-Reported Outcomes (PROs) and Patient-Reported Outcome Measures (PROMs). Health Serv Insights. 2013;4(6):61-68.

14. Lipscomb J, Gotay CC, Snyder CF. Patient-reported outcomes in cancer: a review of recent research and policy initiatives. $C A$ Cancer J Clin. 2007;57(5):278-300.

15. Cano SJ, Klassen A, Pusic AL. The science behind quality-of-life measurement: a primer for plastic surgeons. Plast Reconstr Surg. 2009;123(3):98e-106e.

16. Black N. Patient reported outcome measures could help transform healthcare. BMJ. 2013;28(346):f167.

17. Pusic AL, Klassen AF, Scott AM, Klok JA, Cordeiro PG, Cano SJ. Development of a new patient-reported outcome measure for breast surgery: the BREAST-Q. Plast Reconstr Surg. 2009;124(2):345-353.

18. Pusic AL, Chen CM, Cano S, et al. Measuring quality of life in cosmetic and reconstructive breast surgery: a systematic review of patient-reported outcomes instruments. Plast Reconstr Surg. 2007;120(4):823-837.

19. Liu L, Branford O, Mehigan S. BREAST-Q measurement of the patient perspective in oncoplastic breast surgery: a systematic review. Plast Reconstr Surg Glob Open. 2018;6(8):e1904.

20. Dean NR, Crittenden T. A five-year experience of measuring clinical effectiveness in a breast reconstruction service using the BREAST-Q patient reported outcomes measure: a cohort study. J Plast Reconstr Aesthet Surg. 2016;69(11):1469-1477.

21. Page MJ, McKenzie JE, Bossuyt PM, et al. The PRISMA 2020 statement: an updated guideline for reporting systematic reviews. BMJ. 2021;29(372):n71.

22. Higgins JP, Savović J, Page MJ, Elbers RG, Sterne JA. Assessing risk of bias in a randomized trial. In: Cochrane Handbookfor Systematic Reviews of Interventions [Internet]. John Wiley \& Sons, Ltd; 2019: 205-228. [cited May 4, 2021]. Available from: https://onlinelibrary.wiley.com/doi/abs/10. 1002/9781119536604.ch8. Accessed November 29, 2021.

23. McGuinness LA, Higgins JPT. Risk-of-bias VISualization (robvis): an R package and Shiny web app for visualizing risk-of-bias assessments. Res Syn Meth. 2021;12(1):55-61.

24. Munn Z, Moola S, Lisy K, Riitano D, Tufanaru C. Methodological guidance for systematic reviews of observational epidemiological studies reporting prevalence and cumulative incidence data. Int J Evid Based Health. 2015;13:147-153. 
25. Qureshi AA, Odom EB, Parikh RP, Myckatyn TM, Tenenbaum MM. Patient-reported outcomes of aesthetics and satisfaction in immediate breast reconstruction after nipple-sparing mastectomy with implants and fat grafting. Aesthet Surg J. 2017;37(9):999-1008.

26. Casella D, Di Taranto G, Onesti MG, Greco M, Ribuffo D. A retrospective comparative analysis of risk factors and outcomes in direct-to-implant and two-stages pre-pectoral breast reconstruction: BMI and radiotherapy as new selection criteria of patients. Eur J Surg Oncol. 2019;45(8):1357-1363.

27. Srinivasa DR, Garvey PB, Qi J, et al. Direct-to-Implant versus two-stage tissue expander/implant reconstruction: 2-year risks and patient-reported outcomes from a prospective, multicenter study. Plast Reconstr Surg. 2017;140(5):869-877.

28. Bennett KG, Qi J, Kim HM, et al. Association of fat grafting with patient-reported outcomes in postmastectomy breast reconstruction. JAMA Surg. 2017;152(10):944-950.

29. Davis GB, Lang JE, Peric M, et al. Breast reconstruction satisfaction rates at a large county hospital. Ann Plast Surg. 2014;72:S61-5.

30. Pirro O, Mestak O, Vindigni V, et al. Comparison of patient-reported outcomes after implant versus autologous tissue breast reconstruction using the BREAST-Q. Plast Reconstr Surg Glob Open. 2017;5(1):e1217.

31. Klifto KM, Aravind P, Major M, et al. Differences between breast cancer reconstruction and institutionally established normative data using the BREAST-Q reconstruction module: a comparative study. Plast Reconstr Surg. 2020;145(6):1371-1379.

32. Santosa KB, Qi J, Kim HM, Hamill JB, Pusic AL, Wilkins EG. Effect of patient age on outcomes in breast reconstruction: results from a multicenter prospective study. J Am Coll Surg. 2016;223 (6):745-754.

33. Reinders FCJ, Young-Afat DA, Batenburg MCT, et al. Higher reconstruction failure and less patient-reported satisfaction after post mastectomy radiotherapy with immediate implant-based breast reconstruction compared to immediate autologous breast reconstruction. Breast Cancer. 2020;27(3):435-444.

34. Santosa KB, Qi J, Kim HM, Hamill JB, Wilkins EG, Pusic AL. Long-term patient-reported outcomes in postmastectomy breast reconstruction. JAMA Surg. 2018;153(10):891-899.

35. Klement KA, Hijjawi JB, LoGiudice JA, Alghoul M, OmesieteAdejare P. Microsurgical breast reconstruction in the obese: a better option than tissue expander/implant reconstruction? Plast. Reconstr Surg. 2019;144(3):539-546.

36. Martinez-López JC, García-Espinoza JA, Flores-Soto D, et al. Patient satisfaction index and quality of life measurement with Breast-Q after breast reconstruction in a plastic surgery center in Mexico. $J$ Biosci Med. 2021;9(6):94-105.

37. Brito ÍM, Fernandes A, Andresen C, Barbosa R, Ribeiro M, Valença-Filipe R. Patient satisfaction with breast reconstruction: how much do timing and surgical technique matter? Eur. $J$ Plast Surg. 2020;43(6):809-818.

38. Rindom MB, Gunnarsson GL, Lautrup MD, et al. Good health-related quality-of-life and high patient-reported satisfaction after delayed breast reconstruction with pedicled flaps from the back. J Plast Reconstr Aesthet Surg. 2021;74(8):1752-1757.

39. Ludolph I, Horch RE, Harlander M, et al. Is there a rationale for autologous breast reconstruction in older patients? A retrospective single center analysis of quality of life, complications and comorbidities after DIEP or ms-TRAM flap using the BREAST-Q. Breast $J$. 2015;21(6):588-595.

40. Zhong T, McCarthy C, Min S, et al. Patient satisfaction and health-related quality of life after autologous tissue breast reconstruction: a prospective analysis of early postoperative outcomes. Cancer. 2012;118(6):1701-1709.

41. Stein MJ, Arnaout A, Lichtenstein JB, et al. A comparison of patient-reported outcomes between Alloderm and Dermacell in immediate alloplastic breast reconstruction: a randomized control trial. J Plast Reconstr Aesthet Surg. 2021;74(1):41-47.
42. Sorkin M, Qi J, Kim HM, et al. Acellular dermal matrix in immediate expander/implant breast reconstruction: a multicenter assessment of risks and benefits. Plast Reconstr Surg. 2017;140(6):1091-1100.

43. Casella D, Di Taranto G, Marcasciano M, et al. Nipple-sparing bilateral prophylactic mastectomy and immediate reconstruction with TiLoop ${ }^{\circledR}$ Bra mesh in BRCA1/2 mutation carriers: a prospective study of long-term and patient reported outcomes using the BREAST-Q. Breast. 2018;39:8-13.

44. Macadam SA, Ho AL, Cook EF Jr, Lennox PA, Pusic AL. Patient satisfaction and health-related quality of life following breast reconstruction: patient-reported outcomes among saline and silicone implant recipients. Plast Reconstr Surg. 2010;125(3):761-771.

45. Caputo GG, Zingaretti N, Kiprianidis I, et al. Quality of Life and early functional evaluation in direct-to-implant breast reconstruction after mastectomy: a comparative study between prepectoral versus dual-plane reconstruction. Clin Breast Cancer. 2021;21(4):344-351.

46. Ghilli M, Mariniello MD, Camilleri V, et al. PROMs in post-mastectomy care: patient self-reports (BREAST-Q ${ }^{\mathrm{TM}}$ ) as a powerful instrument to personalize medical services. Eur J Surg Oncol. 2020;46(6):1034-1040.

47. Sinha S, Ruskin O, D'Angelo A, McCombe D, Morrison WA, Webb A. Are overweight and obese patients who receive autologous free-flap breast reconstruction satisfied with their postoperative outcome? A single-centre study. J Plast Reconstr Aesthet Surg. 2016;69 (1):30-36.

48. Song D, Slater K, Papsdorf M, et al. Autologous breast reconstruction in women older than 65 years versus women younger than 65 years: a multi-center analysis. Ann of Plast Surg. 2016;76(2):155-163.

49. McCarthy CM, Klassen AF, Cano SJ, et al. Patient satisfaction with postmastectomy breast reconstruction: a comparison of saline and silicone implants. Cancer. 2010;116(24):5584-5591.

50. Negenborn VL, Dikmans REG, Bouman M-B, Wilschut JA, Mullender MG, Salzberg CA. Patient-reported outcomes after ADM-assisted implant-based breast reconstruction: a cross-sectional study. Plast Reconstr Surg Glob Open. 2018;6(2):e1654.

51. Cano SJ, Klassen AF, Scott AM, Cordeiro PG, Pusic AL. The BREAST-Q: further validation in independent clinical samples. Plast Reconstr Surg. 2012;129(2):293-302.

52. Fuzesi S, Cano SJ, Klassen AF, Atisha D, Pusic AL. Validation of the electronic version of the BREAST-Q in the army of women study. Breast. 2017;33:44-49.

53. Anagnostopoulos F, Myrgianni S. Body image of Greek breast cancer patients treated with mastectomy or breast conserving surgery. J Clin Psychol Med Settings. 2009;16(4):311-321.

54. Koçan S, Gürsoy A. Body image of women with breast cancer after mastectomy: a qualitative research. $J$ Breast Health. 2016;12 (4):145-150.

55. Duggal CS, Metcalfe D, Sackeyfio R, Carlson GW, Losken A. Patient motivations for choosing postmastectomy breast reconstruction. Ann Plast Surg. 2013;70(5):574-580.

56. Chun YS, Verma K, Rosen H, et al. Implant-based breast reconstruction using acellular dermal matrix and the risk of postoperative complications. Plast Reconstr Surg. 2010;125(2):429-436.

57. Dikmans REG, Negenborn VL, Bouman M-B, et al. Two-stage implant-based breast reconstruction compared with immediate one-stage implant-based breast reconstruction augmented with an acellular dermal matrix: an open-label, Phase 4, multicentre, randomised, controlled trial. The Lancet Oncol. 2017;18(2):251-258.

58. Albornoz CR, Matros E, McCarthy CM, et al. Implant breast reconstruction and radiation: a multicenter analysis of long-term health-related quality of life and satisfaction. Ann Surg Oncol. 2014;21(7):2159-2164.

59. Koppiker CB, Noor AU, Dixit S, et al. Implant-based breast reconstruction with autologous lower dermal sling and radiation therapy outcomes. Indian J Surg. 2019;81(6):543-551. 
60. Rowland JH, Desmond KA, Meyerowitz BE, Belin TR, Wyatt GE, Ganz PA. Role of breast reconstructive surgery in physical and emotional outcomes among breast cancer survivors. $J$ Natl Cancer Inst. 2000;92(17):1422-1429.

61. Negenborn VL, Young-Afat DA, Dikmans REG, et al. Quality of life and patient satisfaction after one-stage implant-based breast reconstruction with an acellular dermal matrix versus two-stage breast reconstruction (BRIOS): primary outcome of a randomised, controlled trial. The Lancet Oncol. 2018;19(9):1205-1214.

62. Gilbert E, Emilee G, Ussher JM, Perz J. Sexuality after breast cancer: a review. Maturitas. 2010;66(4):397-407.

63. Spector DJ, Mayer DK, Knafl K, Pusic A. Women's recovery experiences after breast cancer reconstruction surgery. $J$ of Psychosoc Oncol. 2011;29(6):664-676.

64. O’Connell R, DiMicco R, Khabra K, et al. Initial experience of the BREAST-Q breast-conserving therapy module. Breast Cancer Res Treat. 2016;160(1):79-89.

65. Davies C, Holcombe C, Skillman J, et al. Protocol: protocol for a mixed-method study to inform the feasibility of undertaking a large-scale multicentre study comparing the clinical and patient-reported outcomes of oncoplastic breast conservation as an alternative to mastectomy with or without immediate breast reconstruction in women unsuitable for standard breast-conserving surgery (the ANTHEM Feasibility Study). BMJ Open. 2021;11:4.

66. Potter S, Holcombe C, Ward JA, et al. Development of a core outcome set for research and audit studies in reconstructive breast surgery. Br J Surg. 2015;102(11):1360-1371.

67. Jaensson M, Dahlberg K, Nilsson U. Factors influencing day surgery patients' quality of postoperative recovery and satisfaction with recovery: a narrative review. Perioperative Med. 2019;8(1):3.

68. Berning V, Laupheimer M, Nübling M, Heidegger T. Influence of quality of recovery on patient satisfaction with anaesthesia and surgery: a prospective observational cohort study. Anaesthesia. 2017;72 (9):1088-1096.

69. Chen CM, Cano SJ, Klassen AF, et al. Measuring quality of life in oncologic breast surgery: a systematic review of patient-reported outcome measures: quality of life in breast surgery. Breast $J$. 2010;16(6):587-597.

70. Ong WL, Schouwenburg MG, van Bommel ACM, et al. A standard set of value-based patient-centered outcomes for breast cancer: the International Consortium for Health Outcomes Measurement (ICHOM) Initiative. JAMA Oncol. 2017;3(5):677-685.

71. Lucas RE. Reevaluating the strengths and weaknesses of self- report measures of subjective well-being. In: E. Diener, S. Oishi, \& L. Tay (Eds.). Handbook of Well-Being. Salt Lake City, UT: DEF publishers; 2018:12. doi: nobascholar.com

72. Ranieri J, Fiasca F, Guerra F, Perilli E, Mattei A, Di Giacomo D. Examining the post-operative well-being of women who underwent mammoplasty: a cross-sectional study. Front Psychiatry. 2021;29 (12):645102.

73. Allen RJ, Sobti N, Patel AR, et al. Laterality and patient-reported outcomes following autologous breast reconstruction with free abdominal tissue: an 8-year examination of BREAST-Q data. Plast Reconstr Surg. 2020;146(5):964-975.

74. Koslow S, Pharmer LA, Scott AM, et al. Long-term patient-reported satisfaction after contralateral prophylactic mastectomy and implant reconstruction. Ann Surg Oncol. 2013;20(11):3422-3429.

75. Ménez T, Michot A, Tamburino S, Weigert R, Pinsolle V. Multicenter evaluation of quality of life and patient satisfaction after breast reconstruction, a long-term retrospective study. Ann Chir Plast Esthét Elsevier. 2017;63(2):126-133.

76. Razzano S, Marongiu F, Wade R, Figus A. Optimizing DIEP flap Insetting for immediate unilateral breast reconstruction: a prospective cohort study of patient-reported aesthetic outcomes. Plast Reconstr Surg. 2019;143(2):261e-70e.
77. Hu ES, Pusic AL, Waljee JF, et al. Patient-reported aesthetic satisfaction with breast reconstruction during the long-term survivorship period. Plast Reconstr Surg. 2009;124(1):1-8.

78. Pusic AL, Matros E, Fine N, et al. Patient-reported outcomes 1 year after immediate breast reconstruction: results of the mastectomy reconstruction outcomes consortium study. J Clin Oncol. 2017;35(22):2499-2506.

79. Shekhawat L, Busheri L, Dixit S, Patel C, Dhar U, Koppiker C. Patientreported outcomes following breast reconstruction surgery and therapeutic mammoplasty: prospective evaluation 1-year post-surgery with BREAST-Q questionnaire. Indian J Surg Oncol. 2015;6(4):356-362.

80. Dieterich M, Angres J, Stubert J, Stachs A, Reimer T, Gerber B. Patient-reported outcomes in implant-based breast reconstruction alone or in combination with a titanium-coated polypropylene mesh - a detailed analysis of the BREAST-Q and overview of the literature. Geburtshilfe Frauenheilkd. 2015;75(7):692-701.

81. Sewart E, Turner NL, Conroy EJ, et al. Patient-reported outcomes of immediate implant-based breast reconstruction with and without biological or synthetic mesh. BJS Open. 2021;5(1):zraa063.

82. Eltahir Y, Bosma E, Teixeira N, Werker PMN, de Bock GH. Satisfaction with cosmetic outcomes of breast reconstruction: investigations into the correlation between the patients' Breast-Q outcome and the judgment of panels. JPRAS Open. 2020;12(24):60-70.

83. Ochoa O, Garza III R, Pisano S, et al. Prospective longitudinal patient-reported satisfaction and health-related quality of life following DIEP flap breast reconstruction: relationship with body mass index. Plast Reconstr Surg. 2019;143(6):1589-1600.

84. Spindler N, Ebel F, Briest S, Wallochny S, Langer S. Quality of life after bilateral risk-reducing mastectomy and simultaneous reconstruction using pre-pectoral silicone implants. Patient Prefer Adherence. 2021;13(15):741-750.

85. Tan ML, Idris DB, Teo LW, et al. Validation of EORTC QLQ-C30 and QLQ-BR23 questionnaires in the measurement of quality of life of breast cancer patients in Singapore. Asia Pac J Oncol Nurs. 2014;1(1):22-32.

86. Ou Z, Tang Y, Fu J, Doucette J, Murimi IB. PCN482 patient-reported outcome measures in breast cancer: a systematic review of EORTC QLQ-C30, FACT-B, and EORTC QLQ-BR23 development and validation. Value in Health. 2019;22:S530.

87. Nguyen J, Popovic M, Chow E, et al. EORTC QLQ-BR23 and FACT-B for the assessment of quality of life in patients with breast cancer: a literature review. J Comp Eff Res. 2015;4(2):157-166.

88. Montazeri A, Harirchi I, Vahdani M, et al. The EORTC Breast Cancer-Specific Quality of Life Questionnaire (EORTC QLQ-BR23): translation and Validation study of the Iranian Version. Qual Life Res. 2000;9(2):177-184.

89. Ashing-Giwa K, Rosales M. A cross-cultural validation of patient-reported outcomes measures: a study of breast cancers survivors. Qual Life Res. 2013;22(2):295-308.

90. Ashing-Giwa KT, Padilla GV, Tejero JS, Kim J. Breast cancer survivorship in a multiethnic sample: challenges in recruitment and measurement. Cancer. 2004;101(3):450-465.

91. Ramadhanty Z, Yarso K, Probandari A. Construct validity and reliability of Indonesian Version of RAND SF-36 quality of life questionnaire in breast cancer patients. Indonesian J Cancer. 2019;13:55.

92. Hahn EA, Segawa E, Kaiser K, Cella D, Smith BD. Validation of the Functional Assessment of Cancer Therapy-Breast (FACT-B) quality of life instrument. JCO. 2015;33(15_suppl):e17753-e17753.

93. Brady MJ, Cella DF, Mo F, et al. Reliability and validity of the Functional Assessment of Cancer Therapy-Breast quality-of-life instrument. J Clin Oncol. 1997;15(3):974-986.

94. Bella O, Cocchiara R, de Luca A, et al. Functional Assessment of Cancer Therapy Questionnaire for Breast Cancer (FACT-B+4): Italian version validation. Clin Ter. 2018;169:e151-4.

95. Krishnan L, Stanton AL, Collins CA, Liston VE, Jewell WR. Form or function? Part 2 objective cosmetic and functional correlates of quality of life in women treated with breast-conserving surgical procedures and radiotherapy . Cancer. 2001;91(12):2282-2287. 


\section{Publish your work in this journal}

Breast Cancer - Targets and Therapy is an international, peer-reviewed open access journal focusing on breast cancer research, identification of therapeutic targets and the optimal use of preventative and integrated treatment interventions to achieve improved outcomes, enhanced survival and quality of life for the cancer patient.
The manuscript management system is completely online and includes a very quick and fair peer-review system, which is all easy to use. Visit http://www.dovepress.com/testimonials.php to read real quotes from published authors. 